TRANSACTIONS OF THE

AMERICAN MATHEMATICAL SOCIETY

Volume 361, Number 1, January 2009, Pages 391-405

S 0002-9947(08)04505-4

Article electronically published on August 19, 2008

\title{
A CAPTURE PROBLEM IN BROWNIAN MOTION AND EIGENVALUES OF SPHERICAL DOMAINS
}

\author{
JESSE RATZKIN AND ANDREJS TREIBERGS
}

\begin{abstract}
We resolve a question of Bramson and Griffeath by showing that the expected capture time of four predators pursuing a single prey, all moving by standard Brownian motion on a line, is finite. Our main tool is an eigenvalue estimate for a particular spherical domain, which we obtain by a coning construction and domain perturbation.
\end{abstract}

In this paper we examine the expected capture time of a single prey pursued by $n$ predators, all moving by independent, standard Brownian motion on a line. Bramson and Griffeath BG first considered this problem and estimated the capture time in various circumstances. In particular, they showed that if initially there are predators on both sides of the prey, then the expected capture time is finite. For this reason, we will assume that the initial position of the prey is $x_{0}(0)=1$ and the initial positions of the predators are $x_{1}(0)=\cdots=x_{n}(0)=0$. In this case, BG showed that the expected capture time is infinite for $n=1,2,3$, and conjectured that it is finite for $n \geq 4$ (as indicated by simulations). Li and Shao LS showed that the expected capture time is finite for $n \geq 5$. Using a similar method, we resolve the remaining case by showing that the expected capture time is finite for $n=4$.

Theorem 1. Let $x_{0}(t)$ be pursued by $x_{1}(t), \ldots, x_{n}(t)$, where $x_{0}, \ldots, x_{n}$ are independent, standard Brownian motions on a line, $x_{0}(0)=1$, and $x_{1}(0)=\cdots=$ $x_{n}(0)=0$. Then the expected capture time is finite if and only if $n \geq 4$.

One key difference between Li and Shao's method in [LS] and our method is that they consider a difference process, while we do a geometric splitting in spherical polar coordinates to reduce the dimension of the problem. See Section 2.2 for more details on our dimension reduction.

Our method of proof is based on the following reformulation of the probability problem as an eigenvalue estimate. We denote the position of the prey at time $t$ as $x_{0}(t)$ and the position of the $j$ th predator at time $t$ as $x_{j}(t)$, yielding a Brownian particle $x(t)=\left(x_{0}(t), x_{1}(t), \ldots, x_{n}(t)\right) \in \mathbb{R}^{n+1}$. By our choice of initial conditions, the initial position of this particle is $x(0)=(1,0, \ldots, 0)$. The time of capture is the first exit time of the Brownian particle $x(t)$ from the cone

$$
\mathcal{C}_{n+1}=\left\{\left(x_{0}, x_{1}, \ldots, x_{n}\right) \mid x_{0} \geq x_{j}, j=1, \ldots, n\right\}
$$

Received by the editors June 9, 2005 and, in revised form, February 21, 2007.

2000 Mathematics Subject Classification. Primary 60J65; Secondary 35P15.

(C)2008 American Mathematical Society 
By the work of DeBlassie [DB], the first exit time of a Brownian particle from a cone $C \subset \mathbb{R}^{n+1}$ is governed by the first eigenvalue of the link $D=C \cap \mathbb{S}^{n}$ of the cone $C$ with the unit sphere. Thus one can prove Theorem 1 by finding a good lower bound for the first eigenvalue of the link $\mathcal{D}_{n}=\mathcal{C}_{n+1} \cap \mathbb{S}^{n}$ of the cone $\mathcal{C}_{n+1}$. To our knowledge, none of the standard estimates (such as Cheeger's inequality or the Faber-Krahn inequality) provides a sufficiently sharp lower bound for the eigenvalue. We find an effective lower bound for the first eigenvalue of $\mathcal{D}_{n}$ in two steps: first we use a coning construction to reduce the dimension of $\mathcal{D}_{n}$, and then we perturb the resulting 2-dimensional domain to increase its eigenvalue.

The rest of the paper proceeds as follows. In Section 1 we discuss the geometry of the cone $\mathcal{C}_{n+1}$, its link $\mathcal{D}_{n}$, and related regions. Section 2.1 sketches DeBlassie's argument relating the finiteness of the expected exit time of a Brownian particle from a cone to the first eigenvalue of its link. In Section 2.2 we separate variables on the sphere to relate eigenvalues of a spherical cone to its link. For the reader's convenience, we also include the proof that the expected capture time is infinite for $n=1,2,3$ and finite for $n \geq 5$ in this section. The heart of the paper lies in Section 3, where we use domain perturbation to prove the $n=4$ eigenvalue estimate. Finally, we describe a numerical computation of $\lambda_{1}\left(\mathcal{D}_{3}\right)$ and a lower bound for $\lambda_{1}\left(\mathcal{D}_{4}\right)$ in Section 4 .

\section{Geometry of the cone $\mathcal{C}$}

The cone $\mathcal{C}_{n+1}$ and its spherical angle $\mathcal{D}_{n}$ have much symmetry. First observe that $\mathcal{C}_{n+1}$ contains the line $\mathcal{L}$ spanned by $(1,1, \ldots, 1)$; this is the line where the inequalities $x_{0} \geq x_{j}, j=1, \ldots, n$ are all equalities. Thus we can split $\mathcal{C}_{n+1}$ as a sum of a line and a lower dimensional cone

$$
\mathcal{C}_{n+1}=\mathcal{L} \oplus \mathcal{V}_{n}
$$

where $\mathcal{V}_{n}=\mathcal{L}^{\perp} \cap \mathcal{C}_{n+1}$. Let $\left\{e_{i}\right\}_{i=0, \ldots, n}$ be the standard basis of $\mathbb{R}^{n+1}$. Notice that the $V_{j}:=e_{0}-e_{j}, j=1, \ldots, n$ are orthogonal to $(1,1, \ldots, 1)$, so $V_{1}, \ldots, V_{n}$ provide a basis of a perpendicular subspace $\mathcal{L}^{\perp}$. Let

$$
\mathcal{T}_{n-1}:=\mathcal{V}_{n} \cap \mathbb{S}^{n-1},
$$

where $\mathbb{S}^{n-1}$ is the unit sphere in $\mathcal{L}^{\perp}=\operatorname{span}\left\{V_{j}\right\}$. The domain $\mathcal{D}_{n}$ is a double cone over $\mathcal{T}_{n-1}$. More precisely, let $N$ be one of the intersection points in $\mathbb{S}^{n} \cap \mathcal{L}$ (there are two such points), and let $(r, \theta)$ be polar coordinates in $\mathbb{S}^{n}$, centered at $N$. Then

$$
\mathcal{D}_{n}=\left\{(r, \theta) \mid \theta \in \mathcal{T}_{n-1}, 0 \leq r \leq \pi\right\},
$$

where we have identified $\mathbb{S}^{n-1}$ with the unit sphere in the tangent space $T_{N} \mathbb{S}^{n}$. In Figure 1 we show $\mathcal{T}_{1}$ and $\mathcal{D}_{2}$.

In later sections, we will use a generalization of this type of spherical cone. In general, let $\Omega$ be a domain in the equatorial $\mathbb{S}^{n-1}$ of $\mathbb{S}^{n}$, and let $r_{0} \in(0, \pi]$. Then we define the truncated cone

$$
\mathcal{T C}\left(\Omega, r_{0}\right):=\left\{(r, \theta) \mid \theta \in \Omega, 0 \leq r \leq r_{0}\right\} .
$$

We abbreviate $\mathcal{T C}(\Omega, \pi)=\mathcal{T C}(\Omega)$. In this notation, we have $\mathcal{D}_{n}=\mathcal{T C}\left(\mathcal{T}_{n-1}\right)=$ $\mathcal{T C}\left(\mathcal{T}_{n-1}, \pi\right)$ 


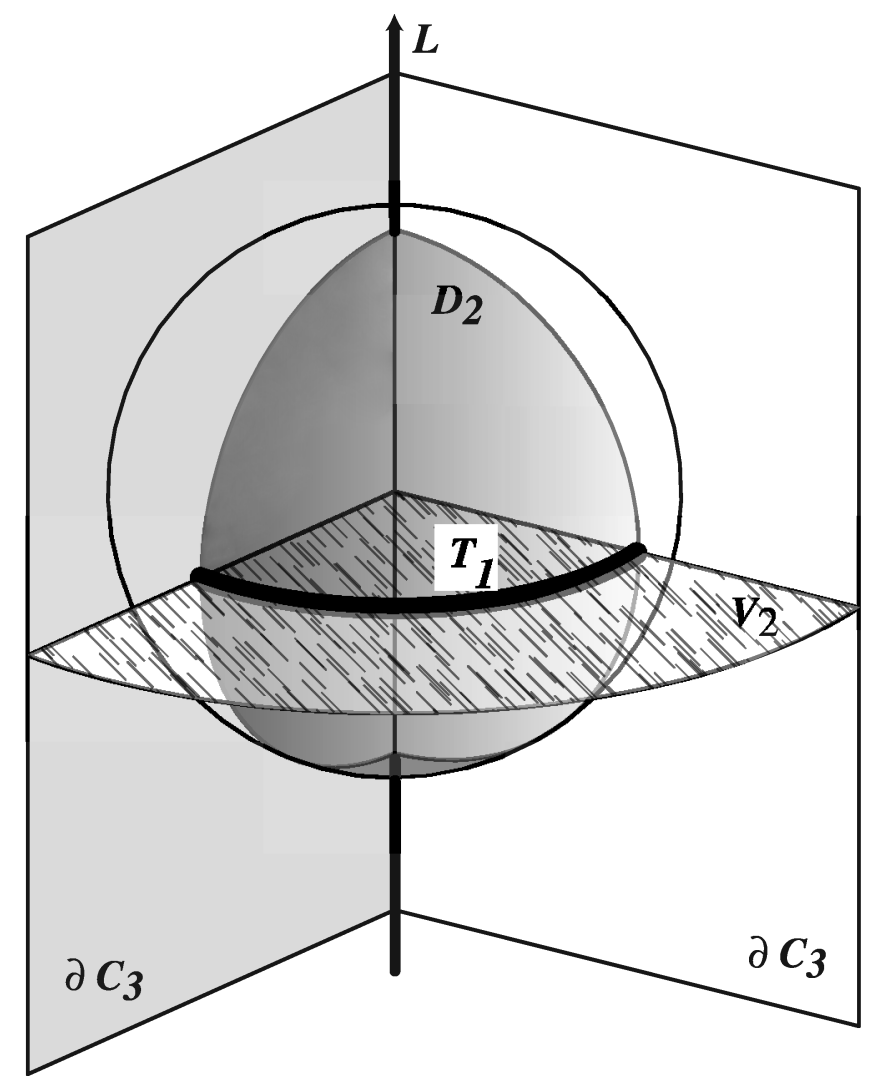

FiguRE 1. This figure shows $\mathcal{T}_{1}$ and $\mathcal{D}_{2}$.

The domain $\mathcal{T}_{n-1}$ has symmetry. If we let

$$
\mathcal{C}_{n+1}^{j}=\left\{\left(x_{0}, x_{1}, \ldots, x_{n}\right) \mid x_{j} \geq x_{k}, j \neq k\right\},
$$

then we see $\mathcal{C}_{n+1}=\mathcal{C}_{n+1}^{0}$. For each pair $j \neq k, \mathcal{C}_{n+1}^{j}$ is congruent to $\mathcal{C}_{n+1}^{k}$ by swapping $x_{j}$ and $x_{k}$. Also they share a common boundary along the $x_{j}=x_{k}$ hyperplane. Thus $\mathcal{T}_{n-1}$ is one of the faces of a regular geodesic tesselation of the standard $\mathbb{S}^{n-1}$ one obtains by connecting the vertices of a regular $(n+1)$-simplex with great circle arcs. In particular, one can compute the diameter of $\mathcal{T}_{n-1}$ as the distance from a vertex to the center of the opposite face, which is

$$
\delta(n-1)=\arccos \left(-\sqrt{\frac{n-1}{2 n}}\right) .
$$

Moreover, the spherical angle of $\mathcal{T}_{n-1}$ at a vertex is $\mathcal{T}_{n-2}$. Thus if $T \subset \mathbb{S}^{n-1}$ is a domain such that $\mathcal{T}_{n-1} \subset T$, then $\mathcal{T}_{n} \subset \mathcal{T C}(T, \delta(n))$ after rotating a vertex to the coordinate center. Thus we can construct a nested sequence of comparison domains $\hat{\mathcal{T}}_{1}, \hat{\mathcal{T}}_{2}, \ldots$ starting with $\mathcal{T}_{1}$ and using the coning construction described above. To this end, we let

$$
\hat{\mathcal{T}}_{1}:=\left[0, \frac{2 \pi}{3}\right]=\mathcal{T}_{1}, \quad \hat{\mathcal{T}}_{n}:=\mathcal{T} \mathcal{C}\left(\hat{\mathcal{T}}_{n-1}, \delta(n)\right) \quad \text { for } n=2,3,4, \ldots
$$


By induction, $\mathcal{T}_{n} \subset \hat{\mathcal{T}}_{n}$. So, by domain montonicity, the first Dirichlet eigenvalues satisfy $\lambda_{1}\left(\mathcal{T}_{n}\right) \geq \lambda_{1}\left(\hat{\mathcal{T}}_{n}\right)$.

\section{Separating VARiables}

We discuss two types of separation of variables in this section. The first type is the separation of variables used in $[\mathrm{DB}$ to estimate expected exit times of Brownian motion from Euclidean cones, and the second is the separation of variables one performs to estimate eigenvalues of a spherical domain with a conical structure.

One needs the domains in question to have a certain amount of regularity in order to separate the variables. In particular, these domains are piecewise $C^{1}$, satisfy an exterior cone condition, and are well approximated (in terms of Hausdorff distance) by finite volume, $C^{1}$ domains. See the introduction to $[\mathrm{DB}]$ for a precise statement. It is straightforward to verify that all of the domains we consider satisfy DeBlassie's hypotheses. We will refer to these domains as nice.

2.1. Separating variables in Euclidean space. The main result of this section is the following lemma:

Lemma 2. The expected capture time for a Brownian prey pursued by $n$ Brownian predators, as described above, is finite if and only if the first eigenvalue of the domain $\mathcal{D}_{n} \subset \mathbb{S}^{n}$ satisfies

$$
\lambda_{1}\left(\mathcal{D}_{n}\right)>2 n+2 .
$$

We will follow $[\mathrm{DB}$ and examine a more general situation than is necessary to prove Lemma 2. Consider a cone $C \subset \mathbb{R}^{n+1}$ over a domain $D \subset \mathbb{S}^{n}$ :

$$
C=\{r \theta \mid r>0, \theta \in D\} .
$$

For a Brownian particle with starting position $x \in C$, let $\tau_{x}$ denote its time of first exit from $C$, and let $u(x, t)=\mathbb{P}\left(\tau_{x}>t\right)$ be the probability that $\tau_{x}>t$. We will see below that $u$ behaves asymptotically (as $t \rightarrow \infty$ ) as follows:

$$
u \sim B_{1} U_{1}\left(\frac{x}{|x|}\right)\left(\frac{|x|^{2}}{2 t}\right)^{a(n)}, \quad 2 a(n)=\left[\left(\frac{n-1}{2}\right)^{2}+\lambda_{1}(D)\right]^{1 / 2}-\frac{n-1}{2} .
$$

Here $B_{1}$ is a constant, and $U_{1}$ is the first eigenfunction on $D$. Notice that DeBlassie's definition of $a_{1}(n)$ is the same as our definition of $2 a(n)$.

The function $u$ satisfies the heat equation

$$
\begin{array}{rlrl}
u_{t} & =\frac{1}{2} \Delta u, & & (x, t) \in C \times(0, \infty) ; \\
u(x, 0) & =1, & x \in \bar{C} ; \\
u(x, t) & =0, & (x, t) \in \partial C \times(0, \infty) .
\end{array}
$$

In polar coordinates, the PDE becomes

$$
2 u_{t}=u_{r r}+\frac{n}{r} u_{r}+\frac{1}{r^{2}} \Delta_{\mathbb{S}^{n}} u .
$$

Moreover, since the solution scales $u(r, \theta)=u\left(\beta r \theta, \beta^{2} t\right)$, we can separate variables and look for a solution of the form $u=R(\xi) U(\theta)$, where $\xi=r^{2} /(2 t)$. Then we get 
a positive separation constant $\lambda_{j}(D)$, and

$$
\lambda_{j}(D)=-\frac{\Delta_{\mathbb{S}^{n}} U}{U}=\frac{4 \xi^{2} \ddot{R}+\left(4 \xi^{2}+2(n+1) \xi\right) \dot{R}}{R} .
$$

The angular equation is

$$
\Delta_{\mathbb{S}^{n}} U+\lambda_{j}(D) U=0
$$

where $\lambda_{j}(D)$ is the $j$-th Dirichlet eigenvalue of $D$. Let

$$
2 a_{j}(n)=\left[\left(\frac{n-1}{2}\right)^{2}+\lambda_{j}(D)\right]^{1 / 2}-\frac{n-1}{2} .
$$

Setting $a=a_{j}$ in (2.2), and letting $R=\xi^{a} \rho(\eta)$ where $\eta=-\xi$, the ODE becomes

$$
\eta \frac{d^{2} \rho}{d \eta^{2}}+\left(2 a+\frac{n+1}{2}-\eta\right) \frac{d \rho}{d \eta}-a \rho=0 .
$$

The solution of this ODE is bounded at zero and has the form

$$
\rho(\xi)={ }_{1} \mathrm{~F}_{1}(a ; 2 a+(n+1) / 2 ;-\xi),
$$

where ${ }_{1} \mathrm{~F}_{1}$ is Kummer's confluent hypergeometric function (see, e.g., $\mathrm{L}$, pp. 260266]). $\rho$ is an entire function because $2 a+(n+1) / 2$ is not zero or a negative integer. Restricting to $K \subset D$ compact and $T>0$, one obtains (see [DB]) an expansion for $u$ of the form

$$
u(x, t)=\sum_{j=1}^{\infty} B_{j}{ }_{1} \mathrm{~F}_{1}\left(a_{j}(n) ; 2 a_{j}(n)+\frac{n+1}{2} ;-\frac{|x|^{2}}{2 t}\right) U_{j}\left(\frac{x}{|x|}\right)\left(\frac{|x|^{2}}{2 t}\right)^{a_{j}(n)} .
$$

This sum converges uniformly in $K \times[T, \infty)$. The leading term (for large $t$ ) in the expansion is $u(x, t) \sim B_{1} U_{1}(x /|x|)\left(|x|^{2} /(2 t)\right)^{a_{1}(n)}$. Thus, the integral $\int_{1}^{\infty} u(x, t) d t$ is finite precisley when $a_{1}>1$, which yields the conclusion of Lemma 2

2.2. Separating variables on the sphere. Next we separate variables on the sphere to relate the eigenvalues $\lambda_{1}\left(\mathcal{D}_{n}\right)$ and $\lambda_{1}\left(\mathcal{T}_{n-1}\right)$. First recall that we can write the Laplacian for $\mathbb{S}^{n}$ in polar coordinates as

$$
\Delta_{\mathbb{S}^{n}} u=u_{r r}+(n-1) \cot r u_{r}+\csc ^{2} r \Delta_{\theta} u,
$$

where $\Delta_{\theta}$ is the Laplacian on the equatorial $\mathbb{S}^{n-1}$. The following two lemmas give us a dimension reduction.

Lemma 3. Let $\Omega$ be a nice domain in an equatorial $\mathbb{S}^{n-1}$ with first eigenvalue $\lambda=\lambda_{1}(\Omega)$, and let $\mathcal{D}=\mathcal{T} \mathcal{C}(\Omega)$ be the double cone over $\Omega$. Then the first Dirichlet eigenvalues of $\mathcal{D}$ and $\Omega$ are related by

$$
\lambda_{1}(\mathcal{D})=\lambda_{1}(\Omega)-\frac{n-2}{2}+\sqrt{\frac{(n-2)^{2}}{4}+\lambda_{1}(\Omega)} .
$$

In particular, $\lambda_{1}(\mathcal{D})>2 n+2$ whenever $\lambda_{1}(\Omega)>2 n$.

Proof. Set $u(r, \theta)=R(r) T(\theta)$, where $R(0)=0=R(\pi)$ and $T(\theta)=0$ for $\theta \in \partial \Omega$. Then $u$ is an eigenfunction on $\mathcal{D}$ with eigenvalue $\mu$ precisely when

$$
T \ddot{R}+(n-1) \cot r T \dot{R}+\csc ^{2} r R \Delta_{\theta} T=-\mu T R .
$$


Separating variables with a positive separation constant $\lambda$ yields

$$
\frac{\sin ^{2} r \ddot{R}+(n-1) \sin r \cos r \dot{R}+\mu \sin ^{2} r R}{R}=\lambda=-\frac{\Delta_{\theta} T}{T} .
$$

Choosing $T$ to be the first eigenfunction of $\Omega$, we obtain the ODE

$$
\sin ^{2} r \ddot{R}+(n-1) \sin r \cos r \dot{R}+\left(\mu \sin ^{2} r-\lambda\right) R=0,
$$

which has regular singular points at $r=0, \pi$. If we try a solution of the form $R=\sin ^{m} r$, for some power $m$ (to be sure the eigenfunction is continuous at the boundary we must have $m>0$ ), we find

$$
\begin{aligned}
0= & m(m-1) \sin ^{m} r \cos ^{2} r-m \sin ^{m+2} r+m(n-1) \sin ^{m} r \cos ^{2} r \\
& +\left(\mu \sin ^{2} r-\lambda\right) \sin ^{m} r \\
= & \sin ^{m} r\left[\left(m^{2}+m(n-2)-\lambda\right) \cos ^{2} r+(\mu-m-\lambda) \sin ^{2} r\right] .
\end{aligned}
$$

Both coefficients must vanish, so we have $\mu=m+\lambda$ and $\lambda=m^{2}+m(n-2)$. Solving for $m$, we find

$$
m=\frac{2-n}{2}+\sqrt{\frac{(2-n)^{2}}{4}+\lambda} .
$$

Next, observe that if $\lambda_{1}(\Omega)=2 n$, then $\lambda_{1}(\mathcal{D})=2 n+2$. Finally, the formula for $\lambda_{1}(\mathcal{D})$ is monotone increasing in $\lambda_{1}(\Omega)$, and so $\lambda_{1}(\mathcal{D})>2 n+2$ whenever $\lambda_{1}(\Omega)>$ $2 n$.

Remark 1. A second solution to equation (2.5) has the form $\sin ^{m} r \cos r$, where $m$ is again given by equation (2.6) but

$$
\mu^{\prime}=\lambda+3 m+n .
$$

This eigenfunction vanishes on $\{\pi / 2\} \times \Omega$, so it corresponds to a higher eigenvalue.

One can generalize the eigenvalue relationship (2.4) to spherical cones of the form $\mathcal{T C}\left(\Omega, r_{0}\right)$, for $0<r_{0}<\pi$, using Gauss's ordinary hypergeometric function (see, e.g., [L, pp. 162-164])

$$
\begin{aligned}
{ }_{2} \mathrm{~F}_{1}(\alpha, \beta ; \gamma ; z)= & 1+\frac{\alpha \beta}{\gamma} \frac{z}{1 !}+\frac{\alpha(\alpha+1) \beta(\beta+1)}{\gamma(\gamma+1)} \frac{z^{2}}{2 !} \\
& +\frac{\alpha(\alpha+1)(\alpha+2) \beta(\beta+1)(\beta+2)}{\gamma(\gamma+1)(\gamma+2)} \frac{z^{2}}{3 !}+\cdots
\end{aligned}
$$

Later we will use the following lemma to relate the eigenvalues of $\hat{\mathcal{T}}_{n-1}$ and $\hat{\mathcal{T}}_{n}$, which we will then estimate to bound the asymptotic decay rates of exit times.

Lemma 4. Let $\Omega$ be a nice proper subdomain in an equatorial $\mathbb{S}^{n-1} \subset \mathbb{S}^{n}$ with eigenvalue $\lambda=\lambda_{1}(\Omega)$. Then the first Dirichlet eigenvalues of $\Omega$ and $\mathcal{T C}\left(\Omega, r_{0}\right)$ are related by

$$
\lambda_{1}\left(\mathcal{T C}\left(\Omega, r_{0}\right)\right)=\mu=\mu\left(n, \lambda, r_{0}\right),
$$


where $\mu$ is the first Dirichlet eigenvalue of the ODE (2.5) on $\left[0, r_{0}\right]$. If $r_{0}>\pi / 2$, then $\mu$ is the unique zero of ${ }_{2} \mathrm{~F}_{1}\left(\alpha_{1}, \beta_{1} ; \gamma_{1} ;(1 / 2)\left(1-\cos r_{0}\right)\right)$ in $(m+\lambda, 3 m+\lambda+n)$, where $m$ is defined in equation (2.6), ${ }_{2} \mathrm{~F}_{1}$ by equation (2.8), and

$$
\begin{aligned}
\alpha_{1}, \beta_{1} & =\frac{1+\sqrt{(n-2)^{2}+4 \lambda} \pm \sqrt{(n-1)^{2}+4 \mu}}{2}, \\
\gamma_{1} & =\frac{2+\sqrt{(n-2)^{2}+4 \lambda}}{2} .
\end{aligned}
$$

If $\mathcal{B}\left(r_{0}\right) \subset \mathbb{S}^{n}$ is the ball of radius $r_{0} \in(0, \pi)$, then

$$
\lambda_{1}\left(\mathcal{B}\left(r_{0}\right)\right)=\mu,
$$

where $\mu=\mu\left(n, r_{0}\right)$ is the first zero of ${ }_{2} \mathrm{~F}_{1}\left(\alpha_{2}, \beta_{2} ; \gamma_{2} ;(1 / 2)\left(1-\cos r_{0}\right)\right)$, where

$$
\alpha_{2}, \beta_{2}=\frac{n-1 \pm \sqrt{(n-1)^{2}+4 \mu}}{2}, \quad \gamma_{2}=\frac{n}{2} .
$$

If $r_{0}>\pi / 2$, then $\mu$ is the unique zero in $(0, n)$.

Proof. We separate variables and look for a solution of the form $R(r)=\sin ^{m} r u(r)$, with $u(r) \neq 0$ on $\left[0, r_{0}\right)$, but $u\left(r_{0}\right)=0$. Then equation (2.5) becomes

$$
\begin{aligned}
0= & \sin ^{m+2} r \ddot{u}+(2 m+n-1) \sin ^{m+1} r \cos r \dot{u} \\
& +\left[m(m+n-2) \cos ^{2} r+(\mu-m) \sin ^{2} r-\lambda\right] \sin ^{m} r u .
\end{aligned}
$$

Now let $u(r)=y(x)$, where $x=(1 / 2)(1-\cos r)$, which transforms the ODE above into

$$
x(1-x) y^{\prime \prime}+\left(m+\frac{1}{2} n-(2 m+n) x\right) y^{\prime}-(\lambda+m-\mu) y=0 .
$$

The solution to this ODE is the hypergeometric function $y(x)={ }_{2} \mathrm{~F}_{1}(\alpha, \beta ; \gamma ; x)$, with

$$
\begin{aligned}
\alpha, \beta & =\frac{2 m+n-1 \pm \sqrt{(2 m+n-1)^{2}-4 \lambda-4 m+4 \mu}}{2} \\
\gamma & =\frac{2 m+n}{2} .
\end{aligned}
$$

By taking $R\left(r ; n, \lambda, r_{0}\right)=\sin ^{m} r_{2} \mathrm{~F}_{1}(\alpha, \beta, \gamma,(1 / 2)(1-\cos r))$ we choose $\mu$ so that $R\left(r_{0} ; n, \lambda, r_{0}\right)=0$. By domain monotonicity, $\mu$ is a strictly increasing function of $r_{0}$ so that there is one root. By Lemma 3 and equation (2.7), $m+\lambda<\mu<3 m+\lambda+n$.

The eigenvalue of the ball is obtained similarly. The radial part of the eigenfunction of the ball satisfies the same equation, except in this case the angular function $T(\theta)$ is constant and $\lambda=0$.

One can use this lemma to compute $\lambda_{1}\left(\hat{\mathcal{T}}_{n-1}\right)$ iteratively. In this case we start with $\lambda_{1}\left(\hat{\mathcal{T}}_{1}\right)=9 / 4$ and apply equation (2.9), then find a lower bound for $a(n)$ using equation (2.1); this is the first column of the summary table in Figure 2 , which gathers lower bound estimates for $a(n)$ using various methods for the reader's reference. From this table, one can see that $\lambda_{1}\left(\hat{\mathcal{T}}_{3}\right)$ is slightly less than 8 , so $\hat{\mathcal{T}}_{3}$ comes close to, but does not quite provide a comparison domain for proving Theorem 1 . 


\begin{tabular}{|c|c|c|c||c|}
\hline$n$ & $\begin{array}{c}\lambda_{1}\left(\hat{\mathcal{I}}_{n-1}\right) \\
\text { bound for } a(n)\end{array}$ & $\begin{array}{c}\lambda_{1}\left(\mathcal{B}_{n-1}\left(R^{*}\right)\right) \\
\text { bound for } a(n)\end{array}$ & $\begin{array}{c}\lambda_{1}\left(\hat{\mathcal{G}}_{n-1}\right) \\
\text { bound for } a(n)\end{array}$ & $\begin{array}{c}\text { numerical } \lambda_{1}\left(\breve{\mathcal{T}}_{n-1}\right) \\
\text { bound for } a(n)\end{array}$ \\
\hline \hline 2 & 2.25 & 2.25 & & \\
& .75000000 & .75000000 & & \\
\hline 3 & 5.00463581 & 4.93604187 & 5.102 & $5.1590 \ldots$ \\
& .89614957 & .88864414 & .90671950 & .9129 \\
\hline 4 & 7.88404072 & 7.84104544 & 8.00087815 & 8.0692 \\
& .99030540 & .98669478 & 1.00007318 & 1.0057 \\
\hline 5 & 10.77018488 & 10.8876959 & 10.90385120 & 10.9819 \\
& 1.05417466 & 1.06229798 & 1.06341192 & 1.0688 \\
\hline 6 & 13.62031916 & 14.0396033 & 13.76957636 & 13.8567 \\
& 1.09882819 & 1.12365271 & 1.10769877 & 1.1129 \\
\hline
\end{tabular}

Figure 2. Table of theoretical and numerical lower bounds for the eigenvalues and $a(n)$

It was already known that the expected capture time is infinite for $n=1,2,3$ [BG] and finite for $n \geq 5[\mathrm{LS}$. Our methods reproduce these results, and we include the proof below for the reader's convenience.

Lemma 5. The expected capture time for a Brownian prey $x_{0}(t)$, pursued by $n$ Brownian predators $x_{1}(t), \ldots, x_{n}(t)$, with the initial conditions $x_{0}(0)=1, x_{1}(0)=$ $\cdots=x_{n}(0)=0$ is infinite for $n=1,2,3$ and finite for $n \geq 5$.

Proof. Inductively applying equation (2.9) and starting with $\lambda_{1}\left(\hat{\mathcal{T}}_{1}\right)=9 / 4$, shows that $\lambda_{1}\left(\hat{\mathcal{T}}_{n-1}\right)>2 n$ for $n=5,6$. As one can see from the second column of the summary table in Figure 2, Li and Shao's [LS] proof works in our setting as well: If $R^{*}$ is chosen so that the ball has the same volume as the $n$-hedron, $\operatorname{Vol}\left(\mathcal{B}_{n-1}\left(R^{*}\right)\right)=$ $\operatorname{Vol}\left(\mathcal{T}_{n-1}\right)=\operatorname{Vol}\left(\mathbb{S}^{n-1}\right) /(n+1)$, then by the Faber-Krahn inequality on spheres (due to Sperner, see [S], C] $), \lambda_{1}\left(\mathcal{T}_{n-1}\right)>\lambda_{1}\left(\mathcal{B}_{n-1}\left(R^{*}\right)\right.$ ). Observing that $\Delta_{n-1} h+$ $2 n h=0$ for $h=(n-1) x_{0}^{2}-x_{1}^{2}-\cdots-x_{n-1}^{2}$, whose nodal domain is the geodesic ball of radius $\arctan \sqrt{n-1}$, then (using Lemma 4) $\lambda_{1}\left(\mathcal{B}_{n-1}\left(R^{*}\right)\right) \geq 2 n$ whenever $R^{*} \leq \arctan \sqrt{n-1}$, which Li and Shao show holds exactly when $n \geq 5$. This implies the expected capture time is finite for $n \geq 5$.

To show that the expected capture time is infinite for $n=1,2,3$, we must show that $\lambda_{1}\left(\mathcal{D}_{n}\right) \leq 2 n+2$, or, equivalently, $\lambda_{1}\left(\mathcal{T}_{n-1}\right) \leq 2 n$. In the case of $n=1$, we have $\mathcal{D}_{1}=[-3 \pi / 4, \pi / 4]$, and so $\lambda_{1}\left(\mathcal{D}_{1}\right)=1<4$. In the case $n=2$, we have $\mathcal{T}_{1}=[0,2 \pi / 3]$, and so $\lambda_{1}\left(\mathcal{T}_{1}\right)=9 / 4<4$. We cannot compute $\lambda_{1}\left(\mathcal{T}_{2}\right)$ so easily, but we can find a test function to show that $\lambda_{1}\left(\mathcal{T}_{2}\right)<6$. Recall the Rayleigh characterization of the first eigenvalue of a domain $\Omega$ :

$$
\lambda_{1}(\Omega)=\inf _{f \in H_{0}^{1}(\Omega), f \neq 0}\left(\frac{\int_{\Omega}|d f|^{2}}{\int_{\Omega} f^{2}}\right) .
$$

To show that $\lambda_{1}\left(\mathcal{T}_{2}\right)<6$, it suffices to find $f_{0} \in H_{0}^{1}\left(\mathcal{T}_{2}\right)$ so that $\int_{\mathcal{T}_{2}}\left|d f_{0}\right|^{2} / \int_{\mathcal{T}_{2}} f_{0}^{2}<6$. Let

$$
f_{0}(x)=\sin \left(\operatorname{dist}\left(x, \partial \mathcal{T}_{2}\right)\right)
$$


and observe that $\left|d f_{0}\right|^{2}=1-f_{0}^{2}$ off the set of focal points of $\partial \mathcal{T}_{2}$, which is a set of measure zero. A computation shows

$$
\lambda_{1}\left(\mathcal{T}_{2}\right) \leq \frac{\int_{\mathcal{T}_{2}}\left|d f_{0}\right|^{2}}{\int_{\mathcal{T}_{2}} f_{0}^{2}}=\frac{2 \pi+\sqrt{3}}{\pi-\sqrt{3}}<6 .
$$

We shall estimate $\lambda_{1}\left(\mathcal{T}_{2}\right)$ numerically in Section 4

\section{The eigenvalue estimate}

In this section we complete the proof of Theorem 1 First observe that it suffices to show $\lambda_{1}\left(\mathcal{T}_{3}\right)>8$. In fact, it suffices to find a sufficiently large lower bound for $\lambda_{1}\left(\mathcal{T}_{2}\right)$ using the following scheme. We define $\lambda_{c r}$ by

$$
8=\mu\left(3, \lambda_{c r}, \delta(3)\right), \quad \lambda_{c r} \simeq 5.101267527 .
$$

If we construct a domain $\mathcal{G}_{2} \subset \mathbb{S}^{2}$ such that $\mathcal{T}_{2} \subset \mathcal{G}_{2}$ and $\lambda_{1}\left(\mathcal{G}_{2}\right)>\lambda_{c r}$, then by domain monotonicity and equation (2.9),

$$
\lambda_{1}\left(\mathcal{T}_{3}\right) \geq \lambda_{1}\left(\mathcal{T} \mathcal{C}\left(\mathcal{T}_{2}, \delta(3)\right)\right) \geq \lambda_{1}\left(\mathcal{T C}\left(\mathcal{G}_{2}, \delta(3)\right)\right)>8
$$

completing the proof of Theorem 1. As the last step in our proof, we construct a domain $\mathcal{G}_{2} \subset \mathbb{S}^{2}$ as a perturbation of $\mathcal{T}_{2}$, such that $\mathcal{T}_{2} \subset \mathcal{G}_{2}$ and $\lambda_{1}\left(\mathcal{G}_{2}\right)=5.102>$ $\lambda_{c r}$.

Figure 3 shows sketches of the domains $\mathcal{T}_{2}, \mathcal{G}_{2}$ and $\hat{\mathcal{T}}_{2}$, after stereographic projection to the plane.

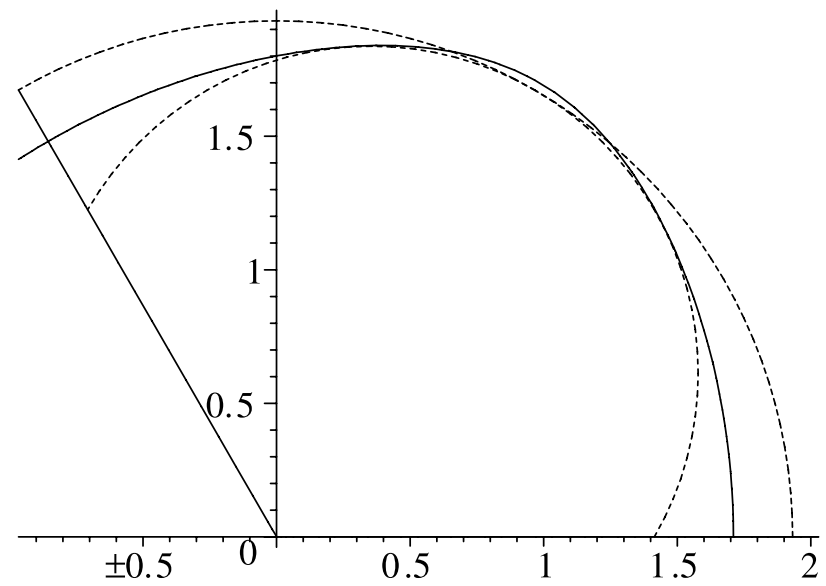

Figure 3 . The inner dashed curve is $\mathcal{T}_{2}$, the solid curve is $\mathcal{G}_{2}$, and the outer dashed curve is $\hat{\mathcal{T}}_{2}$. We generated this figure using the computer program MAPLE.

Previous work of Rayleigh $[\mathrm{R}$ and Pólya-Szegő [PS] motivates us to consider this type of domain perturbation. They studied the eigenvalue of a planar domain which has the form $\{(r, \theta) \mid 0 \leq r \leq c+\epsilon f(\theta)\}$ in polar coordinates, for some small $\epsilon>0$, and gave an expression for the eigenvalue $\lambda_{1}$ in terms of $\epsilon f$. In our case, we fix $\lambda=\lambda_{1}$ and find a domain $\mathcal{G}_{2}$ with $\lambda$ as its first eigenvalue. 
Suppose the functions $R(r)$ and $\Theta(\theta)$ satisfy

$$
\begin{aligned}
\Theta^{\prime \prime}+\lambda \Theta & =0, \quad 0 \leq \theta \leq \frac{2 \pi}{3} ; \\
\Theta(0) & =0 ; \\
\Theta(2 \pi / 3) & =0 ; \\
\sin ^{2} r \ddot{R}+\sin r \cos r \dot{R}+\left(\mu \sin ^{2} r-\lambda\right) R & =0 ; \quad 0 \leq r<\pi ; \\
R(0) & =0 .
\end{aligned}
$$

Then $u(r, \theta)=R(r) \Theta(\theta)$ is the first eigenfunction of $\Delta_{\mathbb{S}^{2}}$ on its nodal domain. By construction, $\Delta_{\mathbb{S}^{2}} u+\mu u=0$, so $u$ is an eigenfunction. Also, $u$ does not change sign on its nodal domain, so it must be the first eigenfunction. In polar coordinates, $u(r, 0)=0=u(r, 2 \pi / 3)$. Let $m=\sqrt{\lambda}$ and set $R(r)=\sin ^{m} r u(r)$, so that equation (2.5) becomes

$$
\sin ^{2}(r) \ddot{u}+(1+2 m) \sin (r) \cos (r) \dot{u}+(\mu-m-\lambda) u=0 .
$$

Next we take $\lambda=9 l^{2} / 4$, corresponding to the $l^{\text {th }}$ mode of the interval $[0,2 \pi / 3]$, and write the solution in terms of the hypergeometric function:

$$
u_{l}(r)={ }_{2} \mathrm{~F}_{1}(3 l / 2+.5+\sqrt{1 / 4+\mu}, 3 l / 2+.5-\sqrt{1 / 4+\mu} ; 1+3 l / 2 ;(1-\cos r) / 2) .
$$

Finally, we take the $\mu=5.102$ superposition of the $l=1,3$ modes to define $H(r, \theta)$ implicitly by the equation

$$
\begin{aligned}
\sin ^{3 / 2}(r) \sin (3 \theta / 2) H(r, \theta):= & (\sin r)^{3 / 2} u_{1}(r) \sin (3 \theta / 2) \\
& -.0003(\sin r)^{9 / 2} u_{3}(r) \sin (9 \theta / 4)
\end{aligned}
$$

and let $\mathcal{G}_{2}$ be the nodal domain of $\sin ^{3 / 2}(r) \sin (3 \theta / 2) H$. By construction, $\lambda_{1}\left(\mathcal{G}_{2}\right)=$ $5.102>\lambda_{c r}$.

The domains $\mathcal{T}_{2}, \mathcal{G}_{2}$ and $\hat{\mathcal{T}}_{2}$ all have three boundary curves, and two of these boundary curves for each domain lie along the great circle $\operatorname{arcs} \theta=0,2 \pi / 3$. For convenience, we convert to a planar domain using stereographic projection, with the south pole corresponding to $r=0$ in polar coordinates on $\mathbb{S}^{2}$. The relationship between polar coordinates $(r, \theta)$ on $\mathbb{S}^{2}$ and polar coordinates $(\rho, \theta)$ on the plane is $\rho=\tan (r / 2)$. Under stereographic projection, the great circle $\operatorname{arcs} \theta=0,2 \pi / 3$ correspond to rays emanating from the origin at angles $0,2 \pi / 3$. The third boundary curve of $\mathcal{T}_{2}$ is given by the circular arc

$$
\left(\zeta(\theta) \cos \theta-\frac{1}{\sqrt{8}}\right)^{2}+\left(\zeta(\theta)-\sqrt{\frac{3}{2}}\right)^{2}=\frac{3}{2},
$$

which we can rewrite as

$$
\rho=\zeta(\theta)=\frac{\sqrt{2} \cos (\theta-\pi / 3)+\sqrt{2 \cos ^{2}(\theta-\pi / 3)+4}}{2} .
$$

Thus, showing $\mathcal{T}_{2} \subset \mathcal{G}_{2}$ is equivalent to showing $H(r, \theta)>0$ along the arc given by $(\rho=\zeta(\theta), \theta)$ for $0 \leq \theta \leq 2 \pi / 3$.

One can see from Figure 3 that $H>0$ along this third boundary component of $\mathcal{T}_{2}$. One strategy yielding a rigorous proof that $H>0$ is the following. We first evaluate $H$ at a point $\theta_{0}$ on the curve, checking $H>0$ at $\left(\rho=\zeta\left(\theta_{0}\right), \theta_{0}\right)$, and bound the derivative $H_{\theta}$ on an interval containing $\theta_{0}$. Our bound $H_{\theta} \geq M$ gives us a lower bound $H>H\left(\theta_{0}\right)-M\left(\theta-\theta_{0}\right)$. Thus $H>0$ on a possibly smaller neighborhood of $\theta_{0}$. We then repeat this procedure with each endpoint of this (smaller) interval. One can simplify the computations by observing $\mathcal{T}_{2}$ and $\mathcal{G}_{2}$ are 
symmetric about the ray $\theta=\pi / 3$. Then it suffices to evaluate $H$ and its derivative at $\theta=0,1 / 2,2 / 3,2 \pi / 9, \pi / 3$.

Given our lower bound $\lambda_{1}\left(\mathcal{T}_{2}\right) \geq \lambda_{1}\left(\mathcal{G}_{2}\right)=5.102$, one can use equation (2.9) to show $\lambda_{1}\left(\mathcal{T}_{3}\right) \geq 8.00087815$. This in turn gives $\lambda_{1}\left(\mathcal{D}_{4}\right) \geq 10.001024501$ and $a(4) \geq 1.00007318$. In the same way, the lower bound $\lambda_{1}\left(\mathcal{T}_{2}\right) \geq 5.102$ gives $a(3) \geq$ .90671950. We summarize our lower bounds for $a(n), n=3,4,5,6$, in the third column of Figure 2, Here the domain $\hat{\mathcal{G}}_{n-1}$ is an iterated cone over $\mathcal{G}_{2}$. Notice that for $n=2$ the domain $\mathcal{T}_{1}$ is 1 -dimensional, so our domain perturbation technique does not apply.

\section{A numerical computation of the first eigenvalue}

In this section we describe a numerical computation approximating the eigenvalue $\lambda_{1}\left(\mathcal{T}_{2}\right)$, which relies on Stenger's sinc-Galerkin scheme [St]. We will show $\lambda_{1}\left(\mathcal{T}_{2}\right) \approx 5.159 \ldots$, and so $a(3) \approx .9128 \ldots$ Also, using equation (2.9), we have $\lambda_{1}\left(\mathcal{T}_{3}\right) \gtrsim 8.0691$ and so $a(4) \gtrsim 1.0057$.

Given $h>0$ and a positive integer $k$, one defines the $k$ th cardinal sinc function of stepsize $h$ as $S(h, k)(h k)=1$ and if $z \neq h k$,

$$
S(h, k)(z):=\frac{\sin (\pi(z-h k) / h)}{\pi(z-h k) / h} .
$$

Following Stenger [St], for $z=x+i y$ we shall let the basis functions be $\phi_{j k}(z)=$ $\alpha_{j}(x) \times \beta_{k}(y)$, where

$$
\alpha_{j}(x)=S(j, h) \circ \ln \left(\frac{x}{\frac{\pi}{2}-x}\right), \quad \alpha_{n+1}(x)=\sin ^{2}(x)-\sum_{l=-n}^{n} \sin ^{2}\left(x_{l}\right) \alpha_{l}(x)
$$

for $j=-n, \ldots, n$ with sinc points $x_{l}=\frac{\pi e^{h l}}{2\left(1+e^{h l}\right)}$ and

$$
\beta_{k}(x)=S(k, h) \circ \log \sinh y, \quad \beta_{n+1}(y)=\operatorname{sech}(y)-\sum_{l=-n}^{n} \operatorname{sech}\left(y_{l}\right) \beta_{l}(y),
$$

for $k=-n, \ldots, n$ with sinc points $y_{l}=\sinh ^{-1}\left(e^{h l}\right)$. We let $x_{n+1}=\pi / 2$ and $y_{n+1}=0$. The dimension of the space of functions $\phi_{j k}$ is $m=(2 n+2)^{2}$. Also $\alpha_{n+1}(\pi / 2)=1$ and $\beta_{n+1}(0)=1$, but both are zero at the other sinc points because their interior sinc approximations are subtracted. The functions $\phi_{j k}=\alpha_{j} \times \beta_{k}$ form a basis of sinc functions when we are working on a rectangular strip.

The most important property of sinc functions is that they are well-suited to approximating integrals in a strip. In particular, one can increase accuracy in a numerical computation by increasing the number of evaluation points, without recomputing grids (as one must do when using a finite element method). In order to take advantage of this simplicity, we will conformally map a subdomain of $\mathcal{T}_{2}$ to a half-infinite strip.

Recall that $\mathcal{T}_{2}$ is an equilateral triangle on $\mathbb{S}^{2}$ with all its interior angles equal to $2 \pi / 3$. Let $T_{1}, T_{2}, T_{3}$ be the vertices of $\mathcal{T}_{2}$, let $S_{j}$ be the midpoint of the side opposite $T_{j}$, and let $F$ be the center of mass of $\mathcal{T}_{2}$. Observe that $\mathcal{T}_{2}$ is invariant under reflection through the lines $F T_{j}$, which divide $\mathcal{T}_{2}$ into six congruent subtriangles (see Figure 44). Thus the first eigenfunction is invariant under these reflections, and we can recover it by restricting to the smaller triangle $\Omega$, which has vertices 


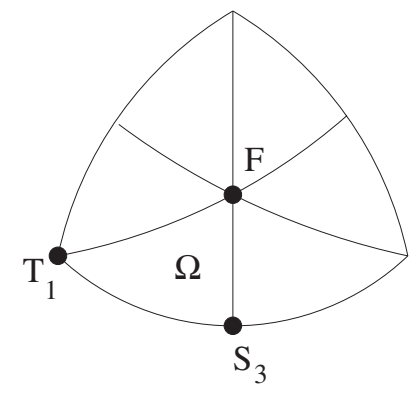

Figure 4 . The three marked points are $F, T_{1}$ and $S_{3}$.

$F, T_{1}, S_{3}$. The triangle $\Omega$ has a right angle at the vertex $S_{3}$, and an angle of $\pi / 3$ at the vertices $F$ and $T_{1}$. The first Dirichlet eigenfunction of $\mathcal{T}_{2}$, restricted to $\Omega$, will have Dirichlet data on the edge $T_{1} S_{3}$ and Neumann data on the edges $F T_{1}$ and $\mathrm{FS}_{3}$.

We first transform $\Omega$ to a planar domain (which we again denote as $\Omega$ ) using stereographic projection, sending $S_{3}$ to 0 . This transformation sends $F$ to $\frac{1}{2}(\sqrt{6}-\sqrt{2})$ and $T_{1}$ to $\frac{i}{2}(\sqrt{6}-\sqrt{2})$. Now $\Omega$ is bounded by the two straight line segments joining 0 to $\frac{1}{2}(\sqrt{6}-\sqrt{2})$ and 0 to $\frac{i}{2}(\sqrt{6}-\sqrt{2})$ and the circular arc joining $\frac{1}{2}(\sqrt{6}-\sqrt{2})$ to $\frac{i}{2}(\sqrt{6}-\sqrt{2})$ which makes an angle of $\pi / 3$ with the axes. Next we find a conformal transformation $f$ which takes $\Omega$ to the half-infinite strip

$$
D=\{z \in \mathbb{C} \mid 0<\Re z<\pi / 2,0<\Im z\}
$$

such that $f(0)=0, f\left(\frac{i}{2}(\sqrt{6}-\sqrt{2})\right)=\pi / 2$, and $f\left(\frac{1}{2}(\sqrt{6}-\sqrt{2})\right)=\infty$. Under this transformation, the first eigenfunction $u$ satisfies

$$
\begin{array}{rlrl}
\Delta^{*} u+\lambda u & =0, & & \text { if } z \in D, \\
u & =0, & & \text { if } \Re \mathrm{e} z=0 \text { and } \Im \mathrm{m} z>0, \\
\frac{\partial u}{\partial n}=0, & & \text { if } 0<\Re \mathrm{e} z<\frac{\pi}{2} \text { and } \Im \mathrm{m} z=0, \text { or if } \Re \mathrm{e} z=\frac{\pi}{2} \text { and } \Im \mathrm{m} z>0,
\end{array}
$$

where $\Delta^{*}=f^{*} \Delta=\Delta_{z}$ is the pulled back Laplacian.

There are formulæ for conformally mapping domains bounded by finitely many circular arcs $[\mathrm{N}]$ generalizing the Schwarz-Christoffel formula. Following Forsythe $\left[\mathrm{F}\right.$, the Schwarz triangle mapping $z \in D$ or from $\sin ^{2} z \in\{\Im z>0\}$ of the upper halfplane to $w \in \Omega$ is given by

$$
\cos ^{2} z=\frac{\left(w^{4}+2 \sqrt{3} w^{2}-1\right)^{3}}{\left(w^{4}-2 \sqrt{3} w^{2}-1\right)^{3}} .
$$

The group generated by reflections of $\Omega$ along its edges in fact tiles the sphere. The inverse function $w \mapsto z^{2}$ extends to a single sheeted cover and is invariant under the symmetry group, thus is a rational function of the plane. Thus we may compute $f$. Writing $g(z)=\cos ^{2 / 3} z$,

$$
f(z)=\sqrt{\frac{1-g}{\sqrt{3}(1+g)+2 \sqrt{1+g+g^{2}}}} .
$$


Pulling back under $w=f(z)$, the conformal weight takes the form

$$
\frac{4\left|\frac{d f}{d z}\right|^{2}}{\left(1+|f|^{2}\right)^{2}}=\frac{\frac{4}{3}\left|\sqrt{3}(1+g)+2 \sqrt{1+g+g^{2}}\right|}{|g|\left(\left|\sqrt{3}(1+g)+2 \sqrt{1+g+g^{2}}\right|+|1-g|\right)^{2}} .
$$

The branch cuts for the square and cube roots may be taken above the negative real axis. Thus $g(D)$ lies in the fourth quadrant, so the denominator in $f$ is nonvanishing.

Let $G\left(z, z^{\prime}\right)$ denote the Green's function for the problem on $D$ :

$$
\begin{array}{rlrl}
\Delta^{*} u & =g, & & \text { if } z \in D, \\
u & =0, & & \text { if } \Re \mathrm{e} z=0 \text { and } \Im \mathrm{m} z>0, \\
\frac{\partial u}{\partial n}=0, & & \text { if } 0<\Re \mathrm{e} z<\pi / 2 \text { and } \Im \mathrm{m} z=0 \text { or if } \Re \mathrm{e} z=\pi / 2 \text { and } \Im \mathrm{m} z>0 .
\end{array}
$$

The Green's function may be found by the method of images. Thus, denoting $w=\sin z=x+i y, w^{*}=-x+y i$ and $\omega=\sin \zeta$, we get $\overline{w^{*}}=(\bar{w})^{*}$. The Green's function is

$$
G(z ; \zeta)=\frac{1}{2 \pi}\left(\ln |w-\omega|-\ln \left|w^{*}-\omega\right|+\ln |\bar{w}-\omega|-\ln \left|\bar{w}^{*}-\omega\right|\right) .
$$

Pulling back by $f$, the eigenvalue problem for the triangle may be restated as finding an eigenvalue for the integral operator

$$
\frac{1}{\lambda} u(z)=-4 \int_{D} \frac{G\left(z, z^{\prime}\right)\left|d f\left(z^{\prime}\right)\right|^{2} u\left(z^{\prime}\right) d z^{\prime}}{\left(1+\left|f\left(z^{\prime}\right)\right|^{2}\right)^{2}}=: \mathcal{A} u(z) .
$$

The key point is to notice that the operator has logarithmic and algebraic singularities at the points $z^{\prime}=0, \pi / 2$ and $z=z^{\prime}$. Thus we need to handle these singularities. The solution is zero along the imaginary axis, but free along the other two sides, which may be extended to functions on the plane which have odd reflection symmetry along the imaginary axis and even reflection symmetry along the other sides. We shall approximate $u(z)$ in an $m$-dimensional space $X_{m}$ with the same symmetries. Also, noticing that the eigenfunction on $\mathcal{T}_{2}$ behaves like $1-\operatorname{dist}^{2}\left(z, T_{2}\right)$ at the vertex $F$, we actually have $u \in \operatorname{Lip}(\bar{D})$ and $u$ decays algebraically at $\infty$. Choosing a basis $\left\{\phi_{1}, \ldots, \phi_{m}\right\}$ of $X_{m}$, we shall compute the matrix of the transformation $A_{\ell k}=\mathcal{P}_{\ell} \mathcal{A} \phi_{k}$, whose largest eigenvalue $\mu_{m} \rightarrow 1 / \lambda$ as $m \rightarrow \infty$ and which has an upper bound $\lambda \leq 1 / \mu_{m}$ [St]. The integral operator shall be computed numerically via sinc quadrature, which can handle such mild singularities.

We make the approximation $u(z) \approx \mathcal{P} u(z)=b^{j k} \phi_{j k}(z)$, where the sums are over $j=-n, \ldots, n+1$ and $k=0, \ldots, n+1$. Here $\phi_{j k}=\alpha_{j} \times \beta_{k}$, where $\alpha_{j}$ and $\beta_{k}$ are defined in equations (4.2) and (4.3), respectively. We define $b^{j k}=\mathcal{P}_{j k} u=$ $u\left(x_{j}+i y_{k}\right)$. Thus the approximation $\mathcal{P} u$ is a collocation, as it equals $u$ at the sinc points. Thus, the matrix is approximated by

$$
\begin{aligned}
A_{j k, p q} & =\int_{D} G\left(x_{j}, y_{k}, \xi, \eta\right) \phi_{p q}(\xi, \eta) \Psi(\xi, \eta) d \xi d \eta \\
& \approx \sum_{\iota, \kappa} v_{\iota} w_{\kappa} G\left(x_{j}, y_{k}, x_{\iota}, y_{\kappa}\right) \phi_{p q}\left(x_{\iota}, y_{\kappa}\right) \Psi\left(x_{\iota}, y_{\kappa}\right),
\end{aligned}
$$


where $\Psi(\xi, \eta)=4\left(1+|f(\xi+i \eta)|^{2}\right)^{-2}|d f(\xi+i \eta)|^{2}$. The approximating sum is carried over $4 m$ terms, corresponding to sinc quadratures in the four regions bounded by singularities (e.g., in case $0<x_{j}<\pi / 2$ and $\left.0<y_{k}\right): D_{I}=\{\xi+i \eta$ : $\left.0<\xi<x_{j}, 0<\eta<y_{k}\right\}, D_{I I}=\left\{\xi+i \eta: x_{j}<\xi<\pi / 2,0<\eta<y_{k}\right\}$, $D_{I I I}=\left\{\xi+i \eta: 0<\xi<x_{j}, y_{k}<\eta\right\}$ and $D_{I V}=\left\{\xi+i \eta: x_{j}<\xi, y_{k}<\eta\right\}$ and where $v_{\iota}$ and $w_{\kappa}$ are corresponding weights and $x_{\iota}$ and $y_{\kappa}$ are corresponding sinc points.

The computation shows that $\lambda_{1}\left(\mathcal{T}_{2}\right) \approx 5.159 \ldots$, which is above the critical value. This is computational evidence that the exit time has finite expectation. We present a table of a few of the computed eigenvalues for approximations in spaces of given dimension, coming from the Sinc-Galerkin collocation scheme described.

\begin{tabular}{ccc}
\cline { 1 - 1 } Dimension & & Eigenvalue Estimate \\
\cline { 1 - 1 } 16 & & 5.948293885960918 \\
1024 & & 5.262319373675790 \\
2116 & & 5.15858593939808193 \\
2304 & & 5.158832705984016 \\
2500 & & 5.158849530710926 \\
2704 & & 5.158968860560663
\end{tabular}

Finally, we can use our numerical computation of $\lambda_{1}\left(\mathcal{T}_{2}\right)$ to find numerical lower bounds for $a(n)$, which we compute in the fourth column of Figure 2 for $n=$ $3,4,5,6$. The domain $\breve{\mathcal{T}}_{n-1}$ is an iterated cone over $\mathcal{T}_{2}$. Again, for $n=2$, the domain $\mathcal{T}_{1}$ is 1 -dimensional, so our numerical scheme does not apply.

\section{ACKNOWLEDGEMENT}

Davar Khoshnevisan told us about this problem. We thank him and Pedro Mendez for lending their ears and expertise to this project. The second author thanks Frank Stenger for suggesting the algorithm of Section 4 .

\section{REFERENCES}

[BG] M. Bramson and D. Griffeath, Capture problems for coupled random walks. in Random Walks, Brownian Motion and Interacting Particle Systems, ed. R. Durrett and H. Kesten, Birkhäuser, 1991. MR1146445 (93e:60192)

[C] I. Chavel, Eigenvalues in Riemannian Geometry. Academic Press, 1984. MR768584 (86g:58140)

[DB] R. D. DeBlassie, Exit times from cones in $\mathbb{R}^{n}$ of Brownian motion. Prob. Theory and Rel. Fields. 74:1-29, 1987. MR863716 (88d:60205)

[F] A. R. Forsythe, Theory of Functions of a Complex Variable, 3rd ed., Dover, 1965, 698700. (Originally published by Cambridge University Press, Cambridge, 1918.) MR0178116 $(31: 2374)$

[L] N. Lebedev, Special Functions and Their Applications. Dover, 1972. (Originally published by Prentice-Hall, Inc., 1965.) MR0350075 (50:2568)

[LS] W. Li and Q.-M. Shao, Capture time of Brownian pursuits. Prob. Theory and Rel. Fields. 121:30-48, 2001. MR:1857107 (2002h:60173)

[N] Z. Nehari, Conformal Mapping. Dover, 1975. (Originally published by McGraw-Hill Book Co., Inc., New York, 1952.) MR0045823 (13:640h)

[PS] G. Pólya and G. Szegö, Isoperimetric Inequalities in Mathematical Physics. Princeton University Press, 1951, 29-30. MR0043486 (13:270d)

[R] Lord Rayleigh (J. W. Strutt), The Theory of Sound (2nd. ed.), Dover, 1945, 336-342. (Originally published by Mcmillan Co., 1894-1896.) MR0016009(7:500e) 
[S] E. Sperner, Zur Symmetrisierung von Funktionen auf Sphären. Math. Z. 134:317-327, 1973. MR0340558 (49:5310)

[St] F. Stenger, Numerical Methods Based on Sinc and Analytic Functions. Springer-Verlag, 1993. MR.1226236 (94k:65003)

Department of Mathematics, University of Connecticut, 196 Auditorium Road, Storrs, ConneCticut 06269

E-mail address: ratzkin@math.uconn.edu

Current address: Department of Mathematics, University of Georgia, Boyd Hall, Athens, Georgia 30602

E-mail address: jratzkin@math.uga.edu

Department of Mathematics, University of Utah, 155 South 1400 East, JWB 233, Salt Lake City, Utah 84112 